\title{
Magnetic resonance imaging of the knee joint in juvenile idiopathic arthritis
}

\author{
Emil Michalski ${ }^{1}$, Monika Ostrowska ${ }^{1}$, Piotr Gietka ${ }^{2}$, Iwona Sudot-Szopińska ${ }^{1}$ \\ 1Department of Radiology, National Institute of Geriatrics, Rheumatology, and Rehabilitation, Warsaw, Poland \\ ${ }^{2}$ Developmental Age Rheumatology Clinic and Polyclinic, National Institute of Geriatrics, Rheumatology, and Rehabilitation, \\ Warsaw, Poland
}

\begin{abstract}
Juvenile idiopathic arthritis (JIA) is an umbrella term for a group of diseases in children younger than 16 years old lasting six weeks or longer. Synovitis may lead to destructive and irreversible joint changes with subsequent functional impairment. Prompt diagnosis is essential to prevent permanent joint damage and preserve joint functionality. In the course of JIA both the axial and peripheral skeleton may be involved in the inflammatory process, but the knee joint is most frequently affected. New drugs and treatment protocols have forced the need for diagnosis at the earliest possible stage. Magnetic resonance imaging (MRI) allows early detection of lesions and constitutes a superior diagnostic imaging method. Synovitis, tenosynovitis, enthesitis, bursitis, osteitis, cartilage loss, bone cysts, and erosions are lesions diagnosed in JIA, and they can be precisely imaged in MRI.

This article aims to present MRI inflammatory features of the knee in children with JIA based on the literature.
\end{abstract}

Key words: children, juvenile idiopathic arthritis, knee, magnetic resonance imaging.

\section{Introduction}

Juvenile idiopathic arthritis (JIA) is a heterogeneous group comprising idiopathic inflammatory arthritis affecting children younger than 16 years of age and lasting six weeks or longer [1]. In western populations, studies have reported an incidence and a prevalence of JIA varying from 2 to 150 per 100,000 [2].

The etiopathogenesis of idiopathic arthritis (IA) is unknown, and the diagnosis is established on the basis of the clinical picture and exclusion, which means that other causes of arthritis need to be ruled out before final diagnosis. Molecular studies indicate many associations between subtypes of JIA and human leukocyte antigen (HLA) genes [3].

Seven subtypes of juvenile idiopathic arthritis have been distinguished by the International League of Associations for Rheumatology (ILAR): systemic JIA, oligoarthritis, polyarthritis positive rheumatoid factor (RF), polyarthritis negative RF, psoriatic arthritis, enthesitis-related arthritis (ERA), and undifferentiated arthritis.
The last subtype does not fit the criteria of any of the other categories, also overlapping syndromes are included into this last subtype. Oligoarthritis subtype is the most common and concerns approximately $27-60 \%$ of children with juvenile idiopathic arthritis [1, 3].

In general, regardless of subtype, JIA is characterized by prolonged synovial inflammation that can cause cartilage and bone damage, and it leads to impairment of physical function and has a significant impact on quality of life [4].

Damaged cartilage facilitates binding of synovial inflammatory cells leading to increased risk of even more cartilage degeneration in the future [5]. Thus, early diagnosis is essential for the prevention of development of potentially irreversible pathological lesions.

Magnetic resonance imaging (MRI) is one of the most advanced imaging techniques available in modern medicine. A wide range of diseases can be diagnosed and monitored in MRI. Rheumatoid disorders primarily affecting the musculoskeletal system are frequent referral causes to radiology departments in order to perform MRI examination. 
Thanks to a lack of ionizing radiation, high spatial and contrast resolution, as well as multiplanar imaging, MRI is a valuable tool in diagnosis of arthritis [6]. It is more sensitive than physical examination, and optimized MRI protocols allow pediatric patients as young as 4 years old to undergo examination without sedation or general anesthesia [6].

The knee is the most commonly affected joint in children with JIA [7], and MRI of this anatomical region is the most frequently performed cross-sectional musculoskeletal imaging examination in the pediatric group of patients [8].

Thus, in this article we review the latest literature concerning MRI of the knee joint in JIA and present the most common MRI findings that every pediatric rheumatologist should be familiar with.

\section{Magnetic resonance imaging - general information}

Magnetic resonance imaging of the knee joints in JIA patients is usually the third imaging examination, after radiography and ultrasonography. Radiography is the standard examination in the assessment of JIA, especially in the differential diagnosis and treatment monitoring of disease.

It is also possible to recognize growth deviations (acceleration or retardation) and easily compare collateral joints, which in some cases may be crucial (for example, to identify early epiphyseal hyperplasia/ballooning due to hyperemia), not mentioning advanced destructive lesion in the knee joint in the course of juvenile idiopathic arthritis [9].

However, this modality is not able to visualize early signs of inflammatory changes in soft tissues, such as synovitis, enthesitis, and tenosynovitis, or bursitis or inflammatory involvement of the bone marrow. Such early findings can be diagnosed with MRI or ultrasonography (US).

Ultrasound is commonly performed for knee joint assessment and provides information on soft tissue involvement (effusions, synovitis, tenosynovitis, bursitis), but it has limited access to all joint surfaces, which means that the hyaline cartilage is only partially visible.

It has also limited ability to evaluate bone tissue, due to lack of penetration of ultrasound through that tissue; therefore, not all subchondral or subcortical cysts and erosions are seen.

The advantage of magnetic resonance imaging over US and radiographs is the ability to precisely assess all tissues involved by JIA, both the soft tissues and bone, including bone marrow edema, which is only visible in MRI and may be an additional feature of early JIA and a predictor of erosive joint damage $[9,10]$.
The added value of ultrasonography is a dynamic examination during joint motion, which provides valuable information (e.g. on the patellofemoral joint), whereas Doppler modalities allow assessment and follow-up of synovium and tenosynovium vascularity [2].

In the detection of early inflammatory lesions in JIA several studies have confirmed that MRI is superior to ultrasonography [11].

Also, in the case of early identification of erosions, Malattia et al. [12] confirmed the superiority of MRI; however, ultrasound was at least as good if not better than plain radiography for the detection of cortical erosions.

Fortunately, due to the availability of efficient treatment protocols in developed western countries, erosive changes in knee joints are rare among patients with JIA nowadays and are seen only in very advanced cases as a late complication [13].

Novel biological drugs have been developed, shifting the focus of pediatric rheumatologists from erosive lesions toward inflammatory changes in the early stages, which cannot be detected in radiography [14].

This has resulted in increased necessity of US and MRI diagnostics of those subtle changes. Prompt diagnosis and treatment are essential to prevent permanent joint damage and preserve joint functionality [2].

\section{Magnetic resonance imaging findings in knee joints in the course of juvenile idiopathic arthritis}

The main indications to knee joint MRI in JIA patients comprise assessment of inflammatory lesions in joints, tendon sheaths, bursae, entheses, inflammatory changes in bone marrow, and their complications (mainly cysts, erosions, cartilage damage).

Synovial thickening, synovial inflammation (synovitis), joint effusions, and bone marrow edema are the most prevalent disease features, followed by destructive, chronic lesions, such as cartilage loss, bone erosions, tendinopathy, and enthesopathy, which occur significantly less frequently [13]. The process is shown in Figures 1-3.

Other findings described by researchers include developmental lesions, such as cruciate ligament thinning as well as menisci hypoplasia due to synovial hypertrophy extending over the meniscal surfaces, which result in meniscal hypoplasia and degradation [15].

Of note, joint complications can result from treatment as well (bone loss and fractures, or avascular necrosis), and MRI is the method of choice in diagnosing the early stages of such pathologies [9].

Table I presents basic lesions that can occur in arthritis, described by the ESSR (the European Society of Mus- 


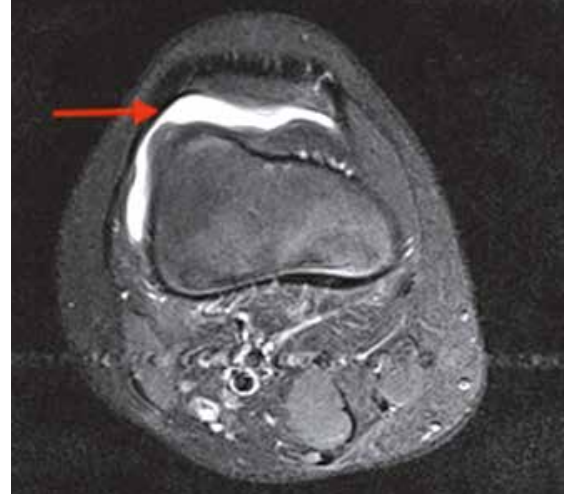

Fig. 1. T2-weighted short-tau inversion recovery magnetic resonance imaging is fluid sensitive sequence. Arrow indicates joint fluid in the axial plane.

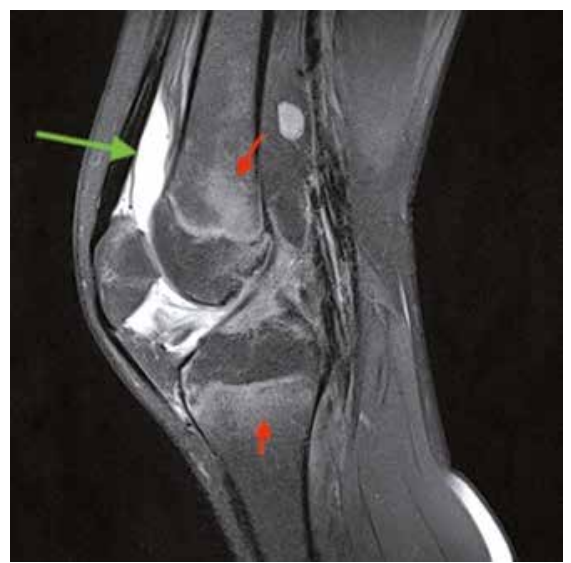

Fig. 2. A 10-year-old boy with advanced juvenile idiopathic arthritis. Non-contrast sagittal proton density fat saturated magnetic resonance imaging shows bone marrow edema (red arrows) and joint effusion (green arrow).

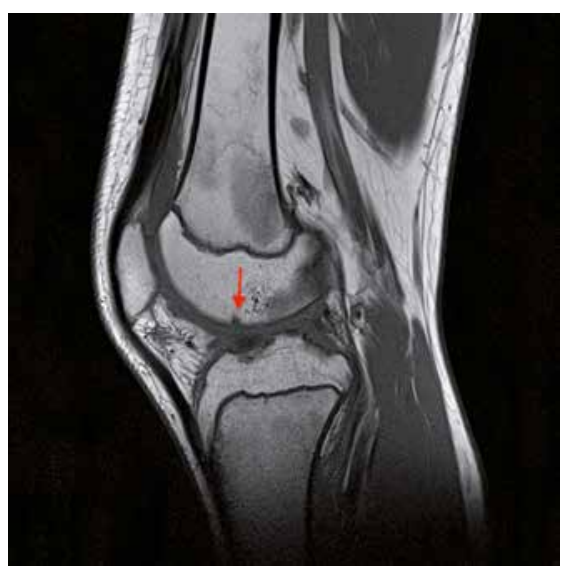

Fig. 3. Non-contrast sagittal proton density knee magnetic resonance imaging of a 12-year-old girl with long history of juvenile idiopathic arthritis presents bone erosion on the articular surface. culoskeletal Radiology) arthritis subcommittee along with their particular MRI definitions [16].

The most commonly seen pathology in the knee joint is synovitis. A vicious circle of inflammatory cells and cytokines interacting with synovium triggers synovial hypertrophy and joint effusion, which are revealed at the early stage.

Subsequently synovitis occurs and progressively leads to cartilage loss and bone erosions. Synovial hypertrophy in MRI according to the Juvenile Idiopathic Arthritis MRI Scoring (JAMRIS) is defined as an enhancing thickened synovial membrane.

Thus, the scoring requires intravenous (i.v.) contrast injection and describes synovitis as enhancing synovial tissue $>2 \mathrm{~mm}$ in thickness [17].

In the subsequent magnetic resonance imaging study of Hemke et al. [18] the maximum measured synovial thickness in knee joints among 57 healthy children was $1.8 \mathrm{~mm}$, which confirmed the good reliability of the $2 \mathrm{~mm}$ cut-off value in the JAMRIS system.

\section{Magnetic resonance imaging protocols and scoring}

According to recommendations of the ESSR arthritis subcommittee, the knee joint protocol should include the following MRI sequences: sagittal proton density (PD) fat suppressed (FS), coronal PD FS, axial PD FS, sagittal or coronal T1-weighted, and T1 or T1 FS after contrast administration [16].

Of course, as the authors underline in the recommendation paper, each MRI protocol should be adjusted to the individual machine scanner specifications, available coils, and image quality in particular sequences.

Hemke et al. [13] proposed the following knee protocol: sagittal and coronal T2-weighted FS or short-tau inversion recovery (STIR), coronal T1-weighted, sagittal gradient echo, or PD and T1-weighted FS post gadolinium sequence. This protocol is able to identify joint effusion, synovial thickening, bone marrow edema and inflammation (osteitis), bone erosions, and cartilage loss [13].

Intravenous contrast injection remains a significant problem, especially in the pediatric population. On the one hand, the accurate assessment of synovitis requires contrast injection because non-contrast MRI reduces sensitivity for detection of inflamed synovium to 62\% [19].

On the other hand, MRI contrast material administration is associated with increased risk of serious adverse events such as allergic reaction, nephrogenic sclerosis, as well as gadolinium brain deposition [20, 21].

For that reason, increased interest in alternative, safer non-contrast sequences has initiated a new direction in research. Diffusion-weighted imaging (DWI) is an 
Table I. Magnetic resonance imaging features of lesions observed in juvenile idiopathic arthritis [16]

\begin{tabular}{|lc|}
\hline Features & Lesions observed in juvenile idiopathic arthritis \\
\hline Joint effusion & $\begin{array}{c}\text { Hyperintense on T2-w and PD images, hypointense on T1-w images and do not enhance immediately } \\
\text { following contrast agent administration only after contrast diffuses to joint fluid from synovium }\end{array}$ \\
\hline Synovitis & $\begin{array}{r}\text { Hyperintense area on T1-w FS sequences following contrast administration. The enhancement within } \\
\text { the synovial membrane should be examined no longer than } 10 \text { minutes after contrast administration. } \\
\text { After this time, the contrast agent permeates into the synovial fluid }\end{array}$ \\
\hline $\begin{array}{l}\text { Bone marrow edema } \\
\text { Enthesitis }\end{array}$ & $\begin{array}{r}\text { Hyperintense area on T2-w and PD images, best visualized by T2 FS or STIR/TIRM sequences, } \\
\text { hypointense on T1-w images, with enhancement following contrast administration }\end{array}$ \\
\hline $\begin{array}{r}\text { Bursitis } \\
\text { on T1-weighted images. The bony part of an enthesis may show bone marrow edema. The soft part } \\
\text { may be thickened. Peri-entheseal tissues may also show features of inflammation }\end{array}$ \\
\hline Intraosseous cysts & $\begin{array}{r}\text { Effusion with the features like above and thickened wall that is hyperintense on T2-w and PD images, } \\
\text { best visualized by T2 FS or STIR/TIRM sequences, hypointense on T1-w images }\end{array}$ \\
\hline Bone erosions & $\begin{array}{c}\text { Sharply marginated trabecular bone defects with disrupted cortical bone continuity, seen in at least } \\
\text { two planes, with low signal intensity on T1-w images }\end{array}$ \\
\hline
\end{tabular}

FS - fat saturated, PD - proton density, STIR - short-tau inversion recovery, T1-W - T1-weighted, T2-W - T2-weighted, TRIM - turbo inversion recovery magnitude.

MRI modality, which does not require contrast and is a promising biomarker in JIA [22].

A recently published study Barendregt et al. [21] confirmed the accuracy of DWI in detecting JIA inflammatory lesions in knee joints and suggested that this modality can even replace contrast-enhanced MRI for imaging of synovial inflammation in children affected by JIA.

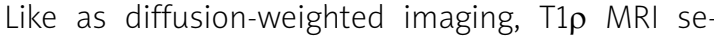
quence does not require contrast administration. It provides cartilage assessment and proved to be acceptable in patients with JIA [23]. However, imaging in DWI sequence requires relevant experience, software, and in everyday radiological practice can be time consuming.

\section{Scoring systems}

In juvenile idiopathic arthritis, disease activity is assessed with scales using clinical examination as well as laboratory tests such as the erythrocyte sedimentation rate (ESR) or C-reactive protein (CRP) [24].

To the best of our knowledge, MRI is still not part of the outcome measures in disease activity, but its potential has been highlighted by the European League Against Rheumatism (EULAR) Paediatric Rheumatology European Society (PReS) imaging task force [25].

In regular clinical practice MRI reporting includes a qualitative approach, which is a description of abnormal findings, their location, number, and size. For treatment monitoring, especially in clinical trials, semiquantitative scorings are proposed.

There are two main magnetic resonance imaging scoring systems evaluating morphological changes, which can be implemented for the knee joint assessment in JIA [26].
First was developed by the International Prophylaxis Study Group (IPSG) and concerned MRI scoring for hemophilic arthropathy [27]. It has been proven to be useful for the evaluation of early and moderate stages of arthropathy and treatment monitoring [28].

Hemke et al. [17] developed the JAMRIS scale for use specifically in JIA, which has been thoroughly researched in recent studies. Compared to the International Prophylaxis Study Group, the JAMRIS system includes bone marrow evaluation but lacks bone cyst assessment and the presence of blood and hemosiderin in the joint (Table II).

Table II. Comparison of features assessed in Juvenile Idiopathic Arthritis MRI Scoring, the International Prophylaxis Study Group and the Combined Juvenile Idiopathic Arthritis Magnetic Resonance Imaging Scoring System

\begin{tabular}{|lccc|}
\hline Feature & JAMRIS & IPSG & Combined JIA \\
\hline Contrast administration & $\mathrm{P}$ & $\mathrm{NP}$ & $\mathrm{P}$ \\
\hline Synovial hypertrophy & + & + & $\mathrm{a}$ \\
\hline Joint effusion & - & + & $\mathrm{b}$ \\
\hline Hemarthrosis & - & + & - \\
\hline Hemosiderin & - & + & - \\
\hline Cartilage lesion & + & + & $\mathrm{b}$ \\
\hline Bone marrow changes & + & - & $\mathrm{a}$ \\
\hline Bone erosion & + & + & $\mathrm{b}$ \\
\hline Subchondral cysts & - & + & - \\
\hline
\end{tabular}

JAMRIS - the Juvenile Idiopathic Arthritis MRI Scoring, JIA - juvenile idiopathic arthritis, IPSG - the International Prophylaxis Study Group, P-performed, NP - not performed, (+)-assessed in scale, $(-)$ - not assessed in scale, a-definition incorporated from the Juvenile Idiopathic Arthritis MRI Scoring, b-definition incorporated from the International Prophylaxis Study Group. 
Table III. Combined juvenile arthritis magnetic resonance imaging scoring system [13]

\begin{tabular}{|c|c|c|}
\hline Feature & Definition & Scoring \\
\hline $\begin{array}{l}\text { Synovial } \\
\text { hypertrophy }\end{array}$ & $\begin{array}{l}\text { An area of the synovial compartment that } \\
\text { shows a thickened synovial membrane } \\
\text { and which can show enhancement after } \\
\text { intravenous gadolinium administration }\end{array}$ & $\begin{array}{c}\text { Scored at six locations }{ }^{\mathrm{a}} \\
\text { (0) normal, } \leq 2 \mathrm{~mm} \text { (1) mild, }>2 \mathrm{~mm} \text { to } \leq 4 \mathrm{~mm} \\
\text { (2) moderate/severe, }>4 \mathrm{~mm} \\
\text { Totals result in a minimum score } \\
\text { of } 0 \text { and a maximum score of } 12\end{array}$ \\
\hline Joint effusion & $\begin{array}{c}\text { An increased amount of fluid within } \\
\text { the synovial compartment with high signal } \\
\text { intensity on T2-w images and low signal } \\
\text { intensity on T1-w images } \\
\text { Joint effusion has no post-gadolinium } \\
\text { enhancement }\end{array}$ & $\begin{array}{l}\text { Scored at the largest pocket of joint effusion: } \\
\text { (0) normal, } \leq 3 \mathrm{~mm} \text { (1) mild, }>3 \mathrm{~mm} \text { to } \leq 5 \mathrm{~mm} \\
\text { (2) moderate/severe, }>5 \mathrm{~mm} \text { large } \\
\text { Totals result in a minimum score of } 0 \\
\text { and a maximum score of } 2\end{array}$ \\
\hline $\begin{array}{l}\text { Cartilage } \\
\text { lesion }\end{array}$ & $\begin{array}{l}\text { Loss of cartilaginous tissue either focally } \\
\text { (superficial or deep) or diffusely }\end{array}$ & $\begin{array}{l}\text { Scored at the most severely affected location: } \\
\text { (0) none, (1) any loss, (2) > 50\% volume loss, } \\
\text { (3) full-thickness loss, } \\
\text { (4) full-thickness loss }>50 \% \text { of surface } \\
\text { Total result in a minimum score of } 0 \\
\text { and a maximum score of } 4\end{array}$ \\
\hline $\begin{array}{l}\text { Bone marrow } \\
\text { changes }\end{array}$ & $\begin{array}{l}\text { An abnormality within the trabecular bone } \\
\text { of the epiphysis, with ill-defined margins } \\
\text { and high signal intensity on T2-w fat-saturated } \\
\text { images and low signal intensity on T1-w }\end{array}$ & $\begin{array}{l}\text { Scored semi-quantitatively based on the subjectively } \\
\text { estimated percentage of involved bone volume at each site } \\
\text { at eight locations }{ }^{\text {b }} \text {, as follows: } \\
\text { (0) none, }(1)<10 \% \text { of the whole bone volume, } \\
(2) \geq 10-25 \% \text { of the whole bone volume, } \\
(3)>25 \% \text { of the whole bone volume } \\
\text { Total result in a minimum score of } 0 \\
\text { and a maximum score of } 24\end{array}$ \\
\hline Bone erosion & $\begin{array}{l}\text { A sharply marginated bone lesion with correct } \\
\text { juxta-articular localization, typical signal } \\
\text { characteristics and visible in two planes with } \\
\text { a cortical break in at least one plane; } \\
\text { on T1-w images there is a loss of the normal low } \\
\text { signal intensity of cortical bone and loss of the } \\
\text { normal high signal intensity of trabecular bone }\end{array}$ & $\begin{array}{l}\text { Scored at the most severely affected: } \\
\text { location (0) none, (1) mild, any loss (2) moderate/severe, } \\
>50 \% \text { surface involvement } \\
\text { Total result in a minimum score } \\
\text { of } 0 \text { and a maximum score of } 2\end{array}$ \\
\hline
\end{tabular}

aPatellofemoral area, suprapatellar recesses, infrapatellar fat pad, adjacent to the anterior and posterior cruciate ligaments, medial posterior condyle, and lateral posterior condyle, blateral patella, medial patella, medial femur condyle, lateral femur condyle, medial weightbearing region of the femur, lateral weight-bearing region of the femur, medial tibia plateau, lateral tibia plateau.

Both scales provide definitions of pathologies [26, 27, 29] and score soft tissue as well as osteochondral parts of the joint. The reliability of Juvenile Idiopathic Arthritis MRI Scoring and JPSG in JIA has been evaluated by the outcome measure in juvenile idiopathic arthritis: outcome measures in rheumatology (OMERACT), with promising results proving their good reliability in knee joint assessment [26].

This study concluded by combining JAMRIS and JPSG into one scale. The combined juvenile arthritis MRI scoring system assesses five features: synovial thickening and bone marrow edema originating from JAMRIS and joint effusion, cartilage loss, and bone erosions originating from JPSG (Table III) [13].

Additional magnetic resonance imaging features that were listed by the authors [26] but were not included in their scoring are as follows: degree of synovial enhancement (scored $0-2)$, infrapatellar fat heterogeneity $(0 / 1$ binary assessment), and the presence of enthesopathy and tendinopathy of the patellar and quadriceps tendons (binary scoring 0/1).

\section{Limitations of magnetic resonance imaging}

Magnetic resonance imaging is not without disadvantages. The procedure is long and requires a motionless position. Very young, agitated patients with additional pain may require participation of anesthesiologists during the MRI scan.

High cost, limited accessibility, and lack of experience in MRI assessment of children affected by JIA are putative causes for underuse of MRI diagnosis in this group of patients. Moreover, the assessment involves one joint, with no possibility of comparing the contralateral side or performing a dynamic examination, which is possible in ultrasound [9, 30]. 
Although enhanced synovial thickening is a crucial MRI finding in JIA, some clinically active patients may show no synovial thickening on MRI of the knee joint [31].

This raises some doubts about the usefulness of MRI in determining whether the disease is active in all JIA patients or the disease activity is overrated in clinical examinations.

On the other hand, the authors did not analyze bone marrow edema in these patients as the possible sole site of disease, without synovitis, and new research is needed to verify which tissues are in fact mostly diseased in knee JIA.

Also, discriminating whether something is normal or pathological is a very challenging task among pediatric patients, and more research is needed in order to establish norms in MRI knee imaging, for example concerning the amount of fluid.

In healthy children usually the largest pockets of fluid are located around the cruciate ligaments and retropatellar region measuring approximately $3 \mathrm{~mm}$ in mean diameter [13].

Scoring systems proposed by IPSG and the combined juvenile arthritis MRI scoring system (shown above) scored abnormal joint effusion as thicker than $3 \mathrm{~mm}$. Also, mild synovial thickening enhancing after contrast injection in the knee joint is not uncommon among children unaffected by clinical arthritis [32].

On the other hand, it is essential to keep in mind that $J I A$ is a medical condition, which in some cases mimics or masks other musculoskeletal diseases [33]. For instance, traumatic injuries, impingements, patellofemoral joint malalignment are underestimated in children, which may cause delay of proper diagnosis.

In 2019 Yousef et al. [34] published a case report on avulsion of quadriceps tendon in an 8-year-old girl affected by JIA after a fall while playing. A distracting history of JIA had been deceiving doctors, which led to misdiagnosis. Consequently, delayed MRI was performed 10 days after trauma, revealing a serious traumatic lesion, which required urgent surgical intervention [34].

Different injury patterns of the knee joint in children than in adults, differences in ligamentous support, normal anatomic variants, and bone marrow appearance are factors that should be taken into consideration when evaluating the knee MRI in children [35].

Another challenging issue in pediatric rheumatology is the definition of remission, both in clinical and imaging studies. Quantitative measurements of synovial volume and the flow in the synovium through dynamic assessment of the uptake of gadolinium contrast can be useful in defining remission [36].

\section{Discussion}

The current criteria for the diagnosis of JIA, disease activity, and identification of remission are based mainly on clinical examination as well as laboratory tests [16]. Clinical assessment, even when performed by an experienced physician, has limited reliability.

Because the knee joint is the most frequently affected in JIA, MRI of this region should be considered as one of the priorities, which can help to diagnose disease at the early stage, tailor appropriate treatment, prevent irreversible progression, and decide when to cease drug administration. Magnetic resonance imaging is a superior and constantly developing musculoskeletal imaging technique.

The literature indicates that it is accurate, reliable, and feasible in detecting and monitoring JIA. Obviously, in clinical practice, the imaging approach usually starts from symmetric knee radiographs in two projections followed by ultrasound.

The former method provides limited data, especially in the early stage of JIA, regarding soft tissue swelling, joint space narrowing, and developmental disorders (mainly early epiphyseal hyperplasia).

Whereas the latter, ultrasound, enriches information with evaluation of synovial inflammation, but information regarding the bone, cartilage, and deeply located soft tissues is limited due to insufficient penetration of ultrasound waves. Nevertheless, it is worth emphasizing that the role of ultrasound in the diagnosis of JIA with knee involvement is indisputable.

Although less readily available, more time consuming, and more costly than ultrasound or radiography, MRI is far more objective, provides quantitative and semiquantitative evaluation of all tissues potentially affected by JIA (both soft and bone), and helps to differentiate from other conditions, such as trauma, overuse, or malignancies.

Quantitative studies are promising, providing more accurate assessment of inflammatory disease activity and treatment monitoring compared with semiquantitative systems [37].

Further research is required, especially regarding scoring systems, non-contrast sequences, and on potential incorporation of MRI into JIA classification criteria, disease activity monitoring, and remission diagnosis.

\section{Conclusions}

Magnetic resonance imaging is, and will undoubtedly continue to be, a method of precise assessment of joint changes in the course of JIA, with the possibility of quantitative assessment. 
Good data archiving allows for a good comparative analysis of changes over time. The refinement of this method of imaging and measures of quantification of these changes may encourage the use of MRI while maintaining its cost-effectiveness.

\section{The authors declare no conflict of interest.}

\section{References}

1. Sudoł-Szopińska I, Eshed I, Jans L, et al. Classifications and imaging of juvenile spondyloarthritis. J Ultrason 2018; 18: 224 233, DOI: $10.15557 /$ jou.2018.0033.

2. Malattia C, Rinaldi M, Martini A. The role of imaging in juvenile idiopathic arthritis. Expert Rev of Clin Immunol 2018; 14: 681 694, DOI: 10.1080/1744666x.2018.1496019.

3. Tugal-Tutkun I, Quartier P, Bodaghi B. Disease of the year: Juvenile idiopathic arthritis-associated uveitis-classification and diagnostic approach. Ocul Immunol Inflamm 2014; 22: 56 63, DOI: 10.3109/09273948.2013.871565.

4. Magni-Manzoni S. Ultrasound in juvenile idiopathic arthritis Pediatr Rheumatol 2016; 14: 33, DOI: 10.1186/s12969-0160096-2.

5. Pap T, Korb-Pap A. Cartilage damage in osteoarthritis and rheumatoid arthritis - two unequal siblings. Nat Rev Rheumato 2015; 11: 606-615, DOI: 10.1038/nrrheum.2015.95.

6. Cellerini M, Salti S, Trapani S, et al. Correlation between clinical and ultrasound assessment of the knee in children with mono-articular or pauci-articular juvenile rheumatoid arthritis. Pediatr Radiol 1999; 29: 117-123, DOI: 10.1007/s002470050554.

7. Hemke R, Nusman CM, van der Heijde DM, et al. Frequency of joint involvement in juvenile idiopathic arthritis during a 5 -year follow-up of newly diagnosed patients: implications for MR imaging as outcome measure. Rheumatol Int 2015; 35: 351-357, DOI: 10.1007/s00296-014-3108-x.

8. Orth RC. The pediatric knee. Pediatr Radiol 2013; 43 (Suppl 1): S90-S98, DOI: 10.1007/s00247-012-2601-1.

9. Sudoł-Szopińska I, Matuszewska G, Gietka P, et al. Imaging of juvenile idiopathic arthritis. Part I: clinical classifications and radiographs. J Ultrason 2016; 16: 225-236, DOI: 10.15557/ jou.2016.0023.

10. Sudoł-Szopińska I, Grochowska E, Gietka P, et al. Imaging of juvenile idiopathic arthritis. Part II: ultrasonography and MRI. J Ultrason 2016; 16: 237-251, DOI: 10.15557/jou.2016.0024.

11. El-Miedany YM, Housny IH, Mansour HM, et al. Ultrasound versus MRI in the evaluation of juvenile idiopathic arthritis of the knee. Joint Bone Spine 2001; 68: 222-230, DOI: 10.1016/S1297319X(01)00269-X.

12. Malattia C, Damasio MB, Magnaguagno F, et al. Magnetic resonance imaging, ultrasonography, and conventional radiography in the assessment of bone erosions in juvenile idiopathic arthritis. Arthritis Rheum 2008; 59: 1764-1772, DOI: 10.1002/art.24313

13. Hemke R, Tzaribachev N, Barendregt AM, et al. Imaging of the knee in juvenile idiopathic arthritis. Pediatr Radiol 2018; 48: 818-827, DOI: 10.1007/s00247-017-4015-6.

14. Damasio MB, Malattia C, Tanturri de Horatio L, et al. MRI of the wrist in juvenile idiopathic arthritis: Proposal of a paediatric synovitis score by a consensus of an international working group. Results of a multicentre reliability study. Pediatr Radiol 2012; 42: 1047-1055, DOI: 10.1007/s00247-012-2392-4.

15. Johnson K. Imaging of juvenile idiopathic arthritis. Pediatr Radiol 2006; 36: 743-758, DOI: 10.1007/s00247-006-0199-x.

16. Sudoł-Szopińska I, Jurik AG, Eshed I, et al. Recommendations of the ESSR Arthritis Subcommittee for the use of magnetic resonance imaging in musculoskeletal rheumatic diseases. Semin Musculoskelet Radiol 2015; 19: 396-411, DOI: 10.1055/s0035-1564696.

17. Hemke R, van Rossum MA, van Veenendaal M, et al. Reliability and responsiveness of the Juvenile Arthritis MRI Scoring (JAMRIS) system for the knee. Eur Radiol 2013; 23: 1075-1083, DOI: 10.1007/s00330-012-2684-y.

18. Hemke R, van den Berg JM, Nusman CM, et al. Contrastenhanced MRI findings of the knee in healthy children; establishing normal values. Eur Radiol 2013; 23: 743-758, DOI: 10.1007/s00330-017-5067-6.

19. Hemke R, Kuijpers TW, van den Berg JM, et al. The diagnostic accuracy of unenhanced MRI in the assessment of joint abnormalities in juvenile idiopathic arthritis. Eur Radiol 2013; 23: 1998-2004, DOI: 10.1007/s00330-013-2770-9.

20. Miller E, Uleryk E, Doria AS. Evidence-based outcomes of studies addressing diagnostic accuracy of MRI of juvenile idiopathic arthritis. Am J Roentgenol 2009; 192: 1209-1218, DOI: 10.2214/AJR.08.2304.

21. Barendregt AM, Mazzoli V, van Gulik EC, et al. Juvenile idiopathic arthritis: diffusion-weighted MRI in the assessment of arthritis in the knee. Radiology 2020; 295: 373-380, DOI: 10.1148/radiol.2020191685.

22. Barendregt AM, van Gulik EC, Lavini C, et al. Diffusion-weighted imaging for assessment of synovial inflammation in juvenile idiopathic arthritis: a promising imaging biomarker as an alternative to gadolinium-based contrast agents. Eur Radiol 2017; 27: 4889-4899, DOI: 10.1007/s00330-017-4876-y.

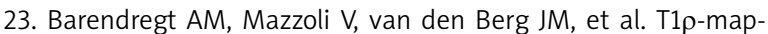
ping for assessing knee joint cartilage in children with juvenile idiopathic arthritis - feasibility and repeatability. Pediatr Radiol 2020; 50: 371-379, DOI: 10.1007/s00247-019-04557-4.

24. Consolaro A, Giancane G, Schiappapietra B, et al. Clinical outcome measures in juvenile idiopathic arthritis. Pediatr Rheumatol Online J 2016; 14: 23, DOI: 10.1186/S12969-016-0085-5.

25. Colebatch-Bourn AN, Edwards CJ, Collado P, et al. EULAR-PReS points to consider for the use of imaging in the diagnosis and management of juvenile idiopathic arthritis in clinical practice. Ann Rheum Dis 2015; 74: 1946-1957, DOI: 10.1136/annrheumdis-2015-207892.

26. Hemke R, Tzaribachev N, Nusman CM, et al. Magnetic resonance imaging (MRI) of the knee as an outcome measure in juvenile idiopathic arthritis: An OMERACT reliability study on MRI scales. J Rheumatol 2017; 44: 1224-1230, DOI: 10.3899/ jrheum.160821.

27. Feldman BM, Funk S, Lundin B, et al. Musculoskeletal measurement tools from the International Prophylaxis Study Group (IPSG). Haemophilia 2008; 14 (Suppl 3): 162-169, DOI: 10.1111/j.1365-2516.2008.01750.x.

28. Lundin B, Manco-Johnson ML, Ignas DM, et al. An MRI scale for assessment of haemophilic arthropathy from the International 
Prophylaxis Study Group. Haemophilia 2012; 18: 962-970, DOI 10.1111/j.1365-2516.2012.02883.x.

29. Doria AS, Keshava SN, Mohanta A, et al. Diagnostic accuracy of ultrasound for assessment of hemophilic arthropathy: MRI correlation. AJR Am J Roentgenol 2015; 204: W336-W347, DOI: 10.2214/AJR.14.12501.

30. Basra HAS, Humphries PD. Juvenile idiopathic arthritis: What is the utility of ultrasound? Br J Radiol 2017; 90: 20160920, DOI: 10.1259/bjr.20160920.

31. van Gulik EC, Hemke R, Welsink-Karssies MM, et al. Normal MRI findings of the knee in patients with clinically active juvenile idiopathic arthritis. Eur J Radiol 2018; 102: 36-40, DOI: 10.1016/j.ejrad.2018.02.027.

32. Nusman CM, Hemke R, Benninga MA, et al. Contrast-enhanced MRI of the knee in children unaffected by clinical arthritis compared to clinically active juvenile idiopathic arthritis patients. Eur Radiol 2016; 26: 1141-1148, DOI: 10.1007/s00330015-3912-z.
33. Greenspan A, Grainger AJ. Articular abnormalities that may mimic arthritis. J Ultrason 2018; 18: 212-223, DOI: $10.15557 /$ jou.2018.0032.

34. Yousef MA, Rosenfeld S. Complete avulsion of quadriceps tendon in a child with juvenile idiopathic arthritis: a case report. JBJS Case Connect 2019; 9: e0195, DOI: 10.2106/JBJS. CC.19.00195.

35. Pai DR, Strouse PJ. MRI of the pediatric knee. AJR Am J Roentgenol 2011; 196: 1019-1027, DOI: 10.2214/AJR.10.6117.

36. Bennett JL, Wood A, Smith N, et al. Can quantitative MRI be used in the clinical setting to quantify the impact of intraarticular glucocorticoid injection on synovial disease activity in juvenile idiopathic arthritis? Pediatr Rheumatol Online J 2019; 17: 74, DOI: 10.1186/s12969-019-0377-7.

37. Boesen M, Kubassova O, Sudoł-Szopińska I, et al. MR Imaging of Joint Infection and Inflammation with Emphasis on Dynamic Contrast-Enhanced MR Imaging. PET Clin 2018; 13: 523-550, DOI: 10.1016/j.cpet.2018.05.007. 